\title{
Slepton flavor nonuniversality, the muon electric dipole moment, and its proposed sensitive search at Brookhaven
}

\author{
Tarek Ibrahim \\ Department of Physics, Faculty of Science, University of Alexandria, Egypt \\ Pran Nath \\ Theoretical Physics Division, CERN CH 1211, Geneva, Switzerland \\ and Department of Physics, Northeastern University, Boston, Massachusetts $02115^{*}$
}

(Received 3 May 2001; published 27 September 2001)

\begin{abstract}
We analyze the electric dipole moment of the electron $\left(d_{e}\right)$, of the neutron $\left(d_{n}\right)$, and of the muon $\left(d_{\mu}\right)$, using the cancellation mechanism in the presence of nonuniversalities of the soft breaking parameters. It is shown that the nonuniversalities in the slepton sector produce a strong violation of the scaling relation $d_{\mu} / d_{e}$ $\simeq m_{\mu} / m_{e}$ in the cancellation region. An analysis of $d_{e}, d_{n}$, and $d_{\mu}$ under the constraints of the current experimental limits on $d_{e}$ and $d_{n}$, and under the constraints of the recent Brookhaven result on $g_{\mu}-2$, shows that in the nonscaling region, $d_{\mu}$ can be as large as $\left(10^{-24}-10^{-23}\right) e \mathrm{~cm}$ and thus within reach of the recently proposed Brookhaven experiment for a sensitive search for $d_{\mu}$ at the level of $10^{-24} e \mathrm{~cm}$.

DOI: 10.1103/PhysRevD.64.093002

PACS number(s): 13.40.Em, 12.60.Jv
\end{abstract}

\section{INTRODUCTION}

Recently a proposal has been made to carry out a dedicated search for the electric dipole moment (EDM) of the muon at the level of $10^{-24} e \mathrm{~cm}[1,2]$. The current experimental limit on the muon EDM from the previous CERN experiment is $d_{\mu}<1.05 \times 10^{-18} e \mathrm{~cm} \mathrm{[3]} \mathrm{and} \mathrm{thus} \mathrm{the} \mathrm{proposed} \mathrm{ex-}$ periment will improve the sensitivity for $d_{\mu}$ by a factor of $10^{5}$ to $10^{6}$ over its previous measurement. As is well known, the electric dipole moment is an important probe of new physics beyond the standard model. Thus the standard model predicts very small values for the EDM's [4], e.g., $d_{e}$ $<10^{-38} e \mathrm{~cm}, d_{\mu}<10^{-35} e \mathrm{~cm}$, and $d_{n}<10^{-31} e \mathrm{~cm}$. However, many models of new physics predict much larger EDM's [5] and thus an increased sensitivity of EDM measurements is a probe of new physics. Supersymmetric (SUSY) models with softly broken supersymmetry are an example of this class of models as they provide abundant new sources of $C P$ violation since the soft breaking parameters can be complex with $C P$ phases $O(1)$. In fact, with $C P$ phases of $O(1)$, an order of magnitude estimate shows that the EDM of the electron and of the neutron are already in excess of the experimental limits which are [6]

$$
d_{e}<4.3 \times 10^{-27} e \mathrm{~cm}, \quad d_{n}<6.3 \times 10^{-26} e \mathrm{~cm} .
$$

Several possible solutions have been offered to resolve this problem. One possibility is to assume that the phases are small which, however, requires a fine tuning [7]. A second possibility is to assume that the sparticles circulating in the loops are heavy, of masses in the range of several $\mathrm{TeV}$, which, however, puts the sparticle spectrum beyond the reach of even the CERN Large Hadron Collider [8]. Another alternative discussed more recently is the possibility of cancellations $[9,10]$ allowing for a satisfaction of the experimen-

\footnotetext{
*Permanent address.
}

tal limits on the electron and on the neutron EDM's with $C P$ phases $O(1)$ and the sparticle spectrum within reach of accelerators. In addition to the above in certain specific unified models, such as in left-right symmetric models, the troublesome phases may automatically be small [11]. Now the normal expectation is that the EDM's scale by the fermion mass and thus the muon EDM should be related to the electron EDM by the scaling relation $d_{\mu} \simeq\left(m_{\mu} / m_{e}\right) d_{e}$. Using the experimental limit on the electron EDM of Eq. (1), this leads to $d_{\mu}<10^{-25} e \mathrm{~cm}$, which is below the sensitivity of the BNL experiment. However, we will show in this paper that the scaling relation $d_{\mu} \simeq\left(m_{\mu} / m_{e}\right) d_{e}$ can be violated strongly in the presence of nonuniversalities. The breakdown of scaling allows significant enhancement of the muon EDM, by as much as a factor of 100 , over the scaling result and $d_{\mu}$ $\sim O\left(10^{-23}\right) e \mathrm{~cm}$ can be gotten, which is well within reach of the proposed Brookhaven experiment.

The analysis presented here is within the framework of the minimal supersymmetric standard model (MSSM). Thus we do not assume any symmetries between the squark and the slepton sector and consequently there are no constraints on the slepton sector arising from limits on flavor changing neutral currents in the quark sector. Further, we do not impose the constraints from $\mu \rightarrow e \gamma$. This sector of the theory involves additional parameters in MSSM and thus no stringent constraints from this sector are expected on the EDM analysis. However, a similar analysis within a specific unified model will require inclusion of such constraints. In addition to the mechanism discussed here, there exist previous analyses where a muon EDM, much larger than what is implied by scaling, can be generated. These consist of analyses in left-right symmetric models [12], in a two Higgs doublet model [13], and in models with certain assumed patterns of textures in the flavor space $[14,15]$. The mechanism we propose in this paper is different. Our technique is a natural extension of the cancellation mechanism using nonuniversalities that are already present within MSSM. 
The outline of the rest of the paper is as follows: In Sec. II we review some possible nonuniversality effects on $d_{e}$ and $d_{\mu}$. In the analysis we impose the constraints on $a_{\mu}^{S U S Y}$ from the BNL experiment [16], as a recent analysis has shown that the BNL result provides stringent constraints on the phases [17]. In Sec. III we give a discussion of the analysis and give results. Conclusions are given in Sec. IV.

\section{SLEPTON NONUNIVERSALITIES, $d_{e}$ AND $d_{\mu}$}

The basic idea we use is that the cancellations arise between the chargino and the neutralino exchange contributions to the EDM of the electron, but such cancellations occur only partially or negligibly for the case of the EDM of the muon. The mechanism that brings this about arises from nonuniversality between the electron and the muon sector. We give now the details of the analysis. First we review some basic formulas that will be relevant in our discussion. The chargino exchange contribution to the charged lepton EDM is proportional to $\kappa_{l} \operatorname{Im}\left(U^{*} V^{*}\right)$ [10], where $\kappa_{l}$ is given by $\kappa_{l}=m_{l} /\left(\sqrt{2} m_{W} \cos \beta\right)$, and $U$ and $V$ are unitary transformations, which diagonalize the chargino mass matrix $M_{C}, U^{*} M_{C} V^{-1}=\operatorname{diag}\left(\tilde{m}_{\chi_{1}^{+}}, \tilde{m}_{\chi_{2}^{+}}\right)$. It is easily seen that the chargino exchange contribution to the EDM of the charged lepton depends on just one combination of the phases, i.e., $\theta_{\mu}+\xi_{2}$, where $\theta_{\mu}$ is the phase of the Higgs mixing parameter $\mu$, and $\xi_{2}$ is the phase of the $S U(2)$ gaugino mass $\tilde{m}_{2}$. The neutralino exchange contribution is proportional to $\operatorname{Im}(\eta)[10]$, where

$$
\begin{aligned}
\eta_{i k}^{l}= & a_{0} c_{0} X_{1 i}^{2} D_{l 1 k}^{*} D_{l 2 k}+b_{0} c_{0} X_{1 i} X_{2 i} D_{l 1 k}^{*} D_{l 2 k} \\
& -\kappa_{l} a_{0} X_{1 i} X_{3 i}\left|D_{l 1 k}\right|^{2}-\kappa_{l} b_{0} X_{2 i} X_{3 i}\left|D_{l 1 k}\right|^{2} \\
& -\kappa_{l} c_{0} X_{1 i} X_{3 i}\left|D_{l 2 k}\right|^{2}+\kappa_{l}^{2} X_{3 i}^{2} D_{l 1 k} D_{l 2 k}^{*} .
\end{aligned}
$$

Here $X$ diagonalizes the neutralino matrix $M_{\chi^{0}}, D_{l}$ diagonalizes the slepton $(\text { mass })^{2}$ matrix, and the constants $a_{0}, b_{0}$, and $c_{0}$ are independent of $A$ parameter and lepton mass. The neutralino exchange contribution depends additionally on the phase combinations $\theta_{\mu}+\xi_{1}$ and $\theta_{\mu}+\alpha_{A_{\tilde{i}}}$. There are several ways in which the nonuniversality between the selectron and the smuon channels can appear. For instance, the sneutrino mass with the $e$ flavor, i.e., $\tilde{\nu}_{e}$ may be different from the sneutrino mass for the $\mu$ flavor, i.e., $\tilde{v}_{\mu}$. In this case if one arranges cancellations between the chargino and the neutralino contributions to occur for $d_{e}$ so that $d_{e}$ is in accord with the current experimental limits, then such exact cancel- lations would not occur for the muon channel due to different chargino contributions, in this case because of the disparity between the masses $m_{\tilde{\nu}_{e}}$ and $m_{\tilde{\nu}_{\mu}}$. A similar situation arises because of the nonuniversality in $M_{L \tilde{e}}^{2}$ and $M_{L \tilde{\mu}}^{2}$ and between $M_{R \tilde{e}}^{2}$ and $M_{R \tilde{\mu}}^{2}$. In this case, if the cancellation is arranged between the chargino and the neutralino for $d_{e}$, it would be upset for $d_{\mu}$ due to neutralino contributions being different between the two cases. There is yet a third possibility in which one may achieve a large cancellation between the chargino and the neutralino contributions for $d_{e}$, but only a partial or no cancellation for $d_{\mu}$. This consists in introducing nonuniversality only in the trilinear soft parameters in the selectron and the smuon channels, i.e., nonuniversalities between $A_{e}$ and $A_{\mu}$. In the following analysis we focus on this type of nonuniversality.

In the analysis we impose the constraints from the recent results from Brookhaven [16] on the size of the new physics contributions to $a_{\mu}=\left(g_{\mu}-2\right) / 2$. This experiment finds a deviation from the standard model result [18] at the $2.6 \sigma$ level so that $[16]$

$$
a_{\mu}^{e x p}-a_{\mu}^{S M}=43(16) \times 10^{-10} .
$$

It has been known for some time that the supersymmetric electroweak contributions can make a significant correction to the muon $g-2$, and the size of this contribution can be as large or larger than the standard model electroweak correction [19-21]. It is also known that contributions to $g-2$ from models with large extra dimensions are not significant and thus do not produce a strong background for supersymmetric contributions [22] (see, however, Ref. [23]). Assuming then that all of the effect of Eq. (3) comes from supersymmetry, one finds that the sparticle spectrum is severely constrained by the BNL result, and further that the sign of $\mu$ in the standard convention [24] with $C P$ invariance is determined to be positive [25]. (Similar conclusions are drawn in Refs. [26-28].) A positive $\mu$ sign is also the one favored by the $b \rightarrow s+\gamma$ constraint [29]. Another important phenomenon is the effect of $C P$ violating phases on $a_{\mu}$. It was shown in Ref. [30] that $a_{\mu}$ is a very sensitive function of the $C P$ phases [30] and that the $C P$ phases can change both the magnitude and the sign of $a_{\mu}$. In the analysis of Ref. [17] this sensitivity was used to constrain the $C P$ phases that enter $a_{\mu}$ by using the constraint of Eq. (3). It was found that as much as $(60 \%-90 \%)$ of the parameter space of $C P$ phases may be eliminated by the BNL constraint [17]. The total contribution to $a_{\mu}$ arises from the chargino and neutralino exchanges where the dominant contribution is from the

TABLE I. EDM's of electron and of neutron for large phases.

\begin{tabular}{lcc}
\hline \hline Case & $\tan \beta, m_{0}, m \frac{1}{2},\left|A_{e}\right|, \xi_{1}, \xi_{2}, \xi_{3}, \theta_{\mu}, \alpha_{A_{e}}$ & $d_{e}, d_{n}(e \mathrm{~cm})$ \\
\hline a & $10,150,281,0.78, .5,-.45, .42, .2,4.1$ & $1.28 \times 10^{-27},-2.04 \times 10^{-26}$ \\
b & $15,120,316,0.77,-.6,-.15,-.64, .3,1.33$ & $3.15 \times 10^{-27},-4.8 \times 10^{-27}$ \\
c & $20,200,246,0.93, .28,-.51,-.11, .4,1.02$ & $-1.33 \times 10^{-27},-1.52 \times 10^{-27}$ \\
d & $20,180,298,0.76,-.6,-.07,-.49, .2,2.6$ & $3.45 \times 10^{-27},-2.24 \times 10^{-27}$ \\
e & $6,100,246,1.31, .4,-.77, .55, .4,1.15$ & $-3.8 \times 10^{-27},-3.9 \times 10^{-26}$ \\
\hline
\end{tabular}


TABLE II. Muon EDM for universal soft parameters.

\begin{tabular}{lcc}
\hline \hline Case & $\left|A_{\mu}\right|, \alpha_{A_{\mu}}$ & $d_{\mu}(e \mathrm{~cm}), a_{\mu}$ \\
\hline $\mathrm{a}$ & $.78,4.1$ & $2.5 \times 10^{-25}, 16.14 \times 10^{-10}$ \\
$\mathrm{~b}$ & $.77,1.33$ & $6.47 \times 10^{-25}, 24.30 \times 10^{-10}$ \\
$\mathrm{c}$ & $.93,1.02$ & $-2.92 \times 10^{-25}, 34.85 \times 10^{-10}$ \\
$\mathrm{~d}$ & $.76,2.6$ & $7.21 \times 10^{-25}, 28.50 \times 10^{-10}$ \\
$\mathrm{e}$ & $1.31,1.15$ & $-7.92 \times 10^{-25}, 13.04 \times 10^{-10}$ \\
\hline
\end{tabular}

chargino exchange [30]. As in the case of the EDM analysis, it is easily seen that the chargino exchange depends on the single phase combination $\theta_{\mu}+\xi_{2}$, while the neutralino contribution depends on several phase combinations, i.e., $\theta_{\mu}$ $+\xi_{i}(i=1.2)$ and on $\theta_{\mu}+\alpha_{A_{\mu}}$. In carrying out the analysis we use the $C P$ dependent formulas for $a_{\mu}$ and impose the constraints of Eqs. (1) and (3) on the phases.

\section{ANALYSIS AND RESULTS}

The general framework of our analysis is MSSM. However, since the parameter space of this model is rather large, we restrict our numerical analysis to a subset of parameters. This subset consists of $m_{0}$ [the universal scalar mass at the grand unified theory (GUT) scale], $m_{1 / 2}$ (magnitude of the universal gaugino mass at the GUT scale), $\left|A_{0}\right|$ (the magnitude of the trilinear coupling of the soft parameters at the GUT scale for all sectors except the smuon sector), and $\tan \beta=\left\langle H_{2}\right\rangle /\left\langle H_{1}\right\rangle$, where $H_{2}$ gives mass to the up quarks and $H_{1}$ gives mass to the down quarks and the leptons. The magnitude of the Higgs mixing parameter $\mu$ is determined by the radiative breaking of the electroweak symmetry. We choose as independent the set of phases $\theta_{\mu}, \xi_{i}(i=1,2,3)$ [the phases of the $U(1), S U(2)$, and $S U(3)$ gauginos] and $\alpha_{A_{0}}$, the phase of $A_{0}$. The trilinear soft parameters $A_{t}, A_{b}$, $A_{e}$, etc., are all evolved from $A_{0}$ using renormalizationgroup equations. Additionally, in the smuon sector, we choose $\left|A_{\mu}\right|$ and $\alpha_{A_{\mu}}$ to be independent parameters to generate the desired nonuniversalities in the smuon sector. The model described above is essentially a supergravity unified model [31] with nonuniversalities [32] in the gaugino sector and in the slepton sector. Our technique consists in first finding a set of soft parameters with large phases, which give a simultaneous cancellation for the case of the EDM of the electron and of the neutron. Nonuniversalities are then used to generate violations of scaling and a large EDM for the muon. In the analysis we include sbottom contributions to

TABLE III. Muon EDM when $\left|A_{\mu}\right|=\left|A_{e}\right|$ and $\alpha_{A_{\mu}} \neq \alpha_{A_{e}}$.

\begin{tabular}{lcc}
\hline \hline Case & $\left|A_{\mu}\right|, \alpha_{A_{\mu}}$ & $d_{\mu}(e \mathrm{~cm}), a_{\mu}$ \\
\hline $\mathrm{a}$ & $.78,-1.5$ & $1.07 \times 10^{-24}, 16.05 \times 10^{-10}$ \\
$\mathrm{~b}$ & $.77,0.0$ & $1.17 \times 10^{-24}, 24.12 \times 10^{-10}$ \\
$\mathrm{c}$ & $.93,0.0$ & $1.51 \times 10^{-24}, 34.80 \times 10^{-10}$ \\
$\mathrm{~d}$ & $.76,-2.0$ & $2.04 \times 10^{-24}, 28.30 \times 10^{-10}$ \\
$\mathrm{e}$ & $1.31,1.8$ & $-1.94 \times 10^{-24}, 13.27 \times 10^{-10}$ \\
\hline \hline
\end{tabular}

TABLE IV. Muon EDM when $A_{\mu} \neq A_{e}$.

\begin{tabular}{lcc}
\hline \hline Case & $\left|A_{\mu}\right|, \alpha_{A_{\mu}}$ & $d_{\mu}(e \mathrm{~cm}), a_{\mu}$ \\
\hline $\mathrm{a}$ & $7.0,1.5$ & $-1.33 \times 10^{-23}, 15.10 \times 10^{-10}$ \\
$\mathrm{~b}$ & $8.0,1.0$ & $-1.27 \times 10^{-23}, 24.28 \times 10^{-10}$ \\
$\mathrm{c}$ & $6.0,-2.0$ & $-1.05 \times 10^{-23}, 35.80 \times 10^{-10}$ \\
$\mathrm{~d}$ & $10.0,-2.5$ & $1.79 \times 10^{-23}, 29.02 \times 10^{-10}$ \\
$\mathrm{e}$ & $7.0,2.5$ & $-1.57 \times 10^{-23}, 14.40 \times 10^{-10}$ \\
\hline \hline
\end{tabular}

the gluonic dimension six operator in the case of the neutron and include two-loop Higgs mediated contributions of the type discussed in Ref. [33]. Regarding the $g-2$ constraint we use a 2 sigma error corridor in Eq. (3) to constrain the sparticle spectrum and the phases, i.e., we use $10.6 \times 10^{-10}$ $<a_{\mu}^{S U S Y}<76.2 \times 10^{-10}$.

In Table I we exhibit five cases, each of which leads to a cancellation in the electron and in the neutron channel producing $d_{e}$ and $d_{n}$ in conformity with the current data, as shown in column 3 of Table I. For the muon case we then use all the same parameters except $A_{\mu}$. We consider three different cases here: (1) universal case where $A_{\mu}=A_{e}$, (2) $\left|A_{\mu}\right|=\left|A_{e}\right|, \quad \alpha_{A_{\mu}} \neq \alpha_{A_{e}}$, and (3) $\left|A_{\mu}\right| \neq\left|A_{e}\right|$ and $\alpha_{A_{\mu}} \neq \alpha_{A_{e}}$. Results of the analysis using these three cases are shown in Tables II, III and IV, respectively. Thus for case 1 one finds that the cancellations between the chargino and the neutralino contributions for $d_{\mu}$, parallel the cancellations of these contributions for $d_{e}$. Thus here a rough scaling results, i.e., $d_{\mu} \sim\left(m_{\mu} / m_{e}\right) d_{e}$ as can be seen by comparing $d_{e}$ of Table I with $d_{\mu}$ of Table II. In this case, the predicted values of $d_{\mu}$ are smaller than what the proposed Brookhaven experiment can probe.

Next we discuss the other two cases, which are more promising because they contain nonuniversality of the soft parameter $\mathrm{A}$, which leads to a breakdown of scaling and allows an enhancement of $d_{\mu}$ over what is allowed by scaling. For case 2, the nonuniversality effects arise from $\alpha_{A_{\mu}}$ $\neq \alpha_{A_{e}}$, and the cancellation between the chargino and the neutralino contributions to $d_{\mu}$ do not parallel the cancellation in $d_{e}$. Because of this one has only a partial cancellation in $d_{\mu}$ in the region of the parameter space where one has a large cancellation for $d_{e}$. This phenomenon leads to a violation of scaling and one obtains here values of $d_{\mu}$ much larger than expected from scaling. The results are exhibited in Table III and one finds that for entries a-e, $d_{\mu}$ is typically larger than its corresponding value in Table II by a factor of $2-3$. Further, all $d_{\mu}$ values listed in Table III fall in the range $O\left(10^{-24}\right) e \mathrm{~cm}$ which is within reach of the proposed Brookhaven experiment [1,2]. Finally we consider case 3, where both the magnitude and the phase of $A_{\mu}$ differ from $A_{e}$. In this case one can manage a much larger value for $d_{\mu}$ over the value expected from scaling. The results are shown in Table IV. In computing $d_{\mu}$ in this case, we have used all the same parameters as in Table I except that the magnitude and the phase of $A_{\mu}$ are treated as independent. As can be seen from Table IV one finds that $d_{\mu}$ can be as large as $O\left(10^{-23}\right) e \mathrm{~cm}$ in this case. For the above three cases the $a_{\mu}$ listed in Tables II, III and IV is consistent with the 2 sigma 


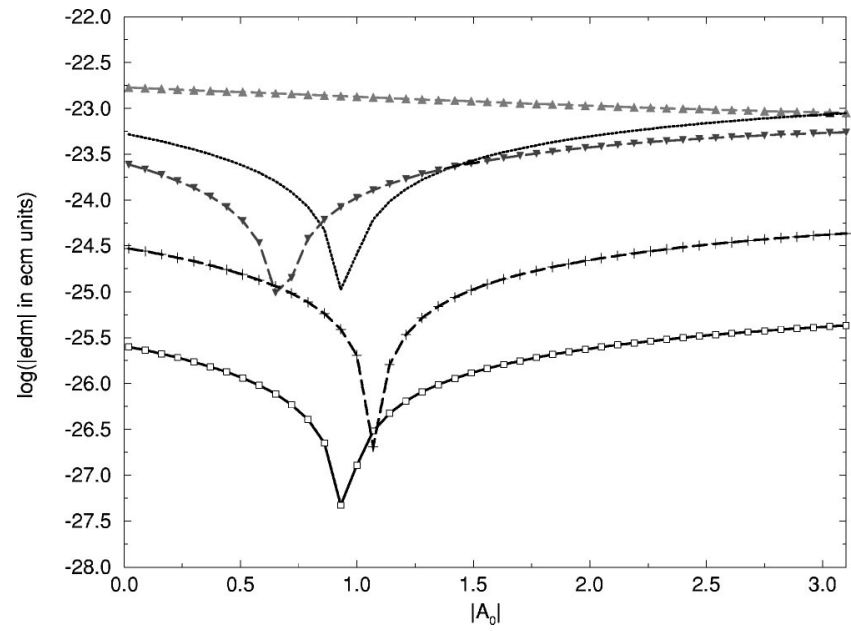

FIG. 1. A plot of the electron EDM $d_{e}$ (solid line with squares), the neutron $\mathrm{EDM} d_{n}$ (dashed line with plus signs), and the muon EDM $d_{\mu}$ (dotted line) as a function of $\left|A_{0}\right|$ for the case when $\tan \beta=10, m_{0}=150, m_{1 / 2}=281, \xi_{1}=.5, \xi_{2}=-.45, \xi_{3}=.42, \theta_{\mu}$ $=.2$, and $\alpha_{A_{e}}=4.1$, where all masses are in $\mathrm{GeV}$ corresponding to case a in Table I. The curve with dashed line with triangles pointed down, is a plot of the muon EDM $d_{\mu}$, which have all the same parameters for $d_{e}$ and $d_{n}$, except that $\alpha_{A_{\mu}}=-1.5$ (corresponding to case a of Table III) and the curve with dashed line with triangles pointed up is a plot of the muon $\operatorname{EDM} d_{\mu}$, which have all the same parameters for $d_{e}$ and $d_{n}$ except that $\left|A_{\mu}\right|=7.0$ and $\alpha_{A_{\mu}}=-1.5$ (corresponding to case a of Table IV).

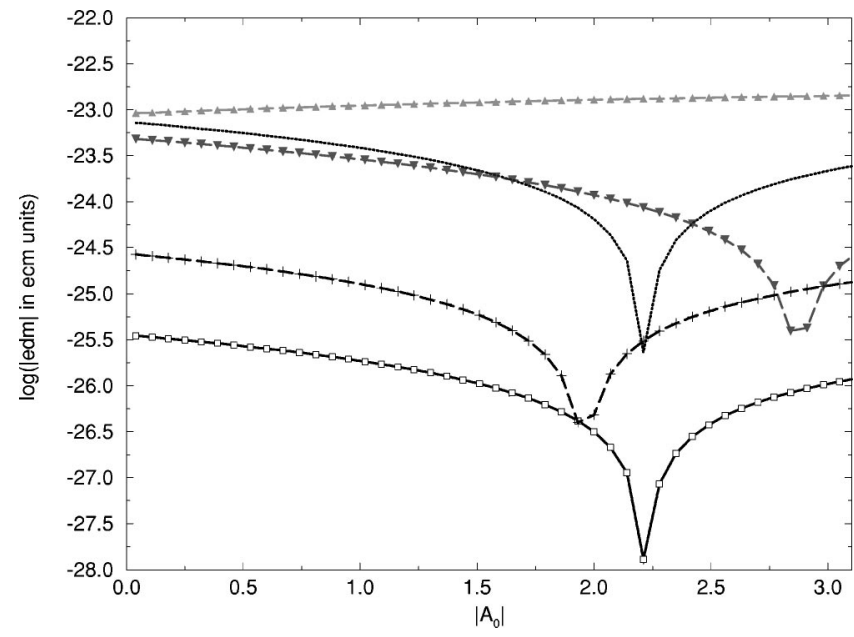

FIG. 2. A plot of the electron EDM $d_{e}$ (solid line with squares), of the neutron EDM $d_{n}$ (dashed line with plus signs), and the muon $\mathrm{EDM} d_{\mu}$ (dotted line) as a function of $\left|A_{0}\right|$ for the case when $\tan \beta=15, m_{0}=120, m_{1 / 2}=316, \xi_{1}=-.6, \xi_{2}=-.15, \xi_{3}=-.64$, $\theta_{\mu}=.3$, and $\alpha_{A_{e}}=1.33$, where all masses are in GeV corresponding to case $\mathrm{b}$ in Table I. The curve with dashed line with triangles pointed down is a plot of the muon $\operatorname{EDM} d_{\mu}$, which have all the same parameters for $d_{e}$ and $d_{n}$ except that $\alpha_{A_{\mu}}=0.0$ (corresponding to case $\mathrm{b}$ of Table III) and the curve with dashed line with triangles pointed up is a plot of the muon EDM $d_{\mu}$, which have all the same parameters for $d_{e}$ and $d_{n}$, except that $\left|A_{\mu}\right|=8.0$ and $\alpha_{A_{\mu}}=1.0$ (corresponding to case $\mathrm{b}$ of Table IV).

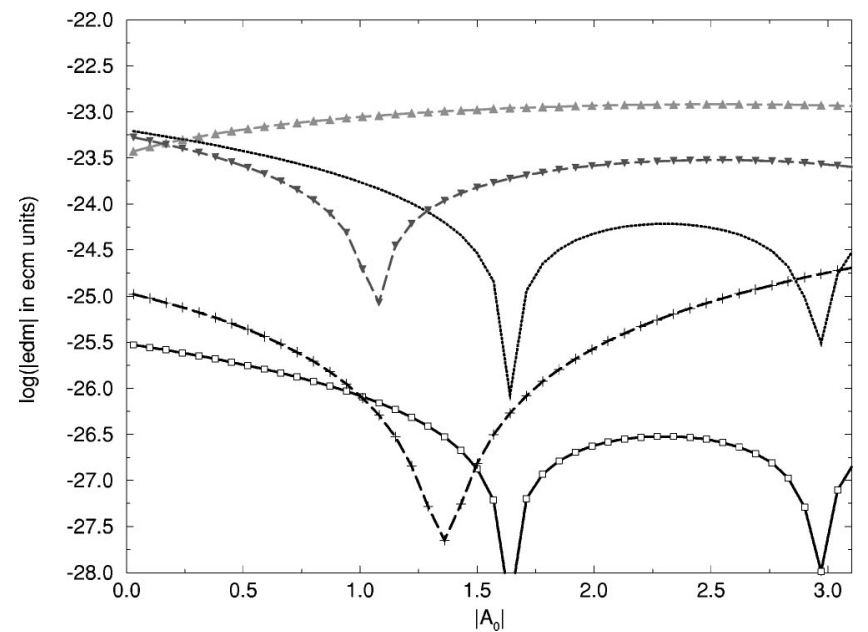

FIG. 3. A plot of the electron EDM $d_{e}$ (solid line with squares), of the neutron EDM $d_{n}$, (dashed line with plus signs), and of the muon $\operatorname{EDM} d_{\mu}$ as a function of $\left|A_{0}\right|$ for the case when $\tan \beta$ $=20, m_{0}=200, m_{1 / 2}=246, \quad \xi_{1}=.28, \quad \xi_{2}=-.51, \quad \xi_{3}=-.11, \quad \theta_{\mu}$ $=.4$, and $\alpha_{A_{e}}=1.02$, where all masses are in $\mathrm{GeV}$ corresponding to case $\mathrm{c}$ in Table I. The curve with dashed line with triangles pointed down is a plot of the muon EDM $d_{\mu}$, which have all the same parameters as for $d_{e}$ and $d_{n}$ except that $\alpha_{A_{\mu}}=0.0$ (corresponding to case $\mathrm{c}$ of Table III) and the curve with dashed line with triangles pointed up is a plot of the muon EDM $d_{\mu}$, which have all the same parameters for $d_{e}$ and $d_{n}$, except that $\left|A_{\mu}\right|=6.0$ and $\alpha_{A_{\mu}}=-2.0$ (corresponding to case $\mathrm{c}$ of Table IV).

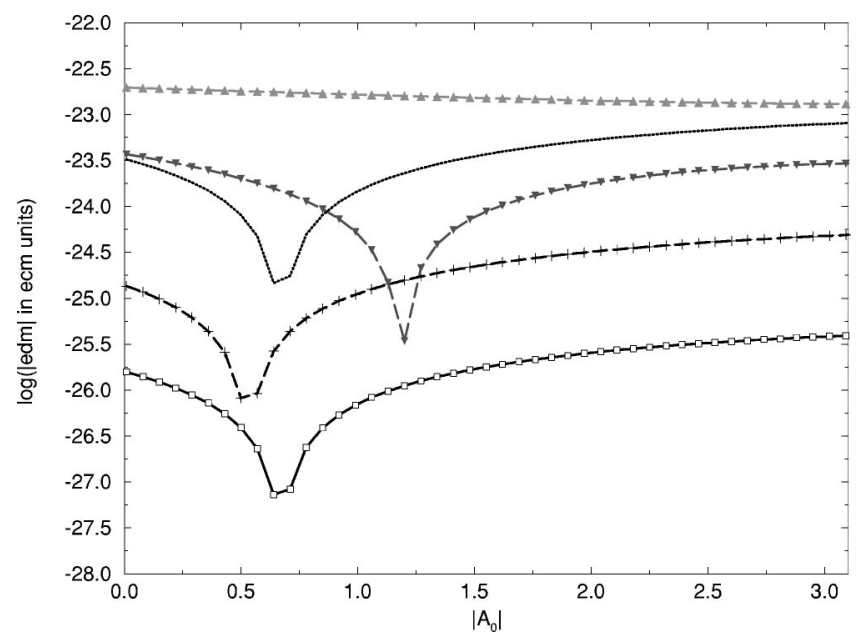

FIG. 4. A plot of the electron EDM $d_{e}$ (solid line with squares), of the neutron EDM $d_{n}$ (dashed line with plus signs), and the muon $\operatorname{EDM} d_{\mu}$, as a function of $\left|A_{0}\right|$ for the case when $\tan \beta=20, m_{0}$ $=180, m_{1 / 2}=298, \xi_{1}=-.6, \quad \xi_{2}=-.07, \quad \xi_{3}=-.49, \theta_{\mu}=.2$, and $\alpha_{A_{e}}=2.6$, where all masses are in $\mathrm{GeV}$ corresponding to case $\mathrm{d}$ in Table I. The curve with dashed line with triangles pointed down is a plot of the muon EDM $d_{\mu}$, which have all the same parameters for $d_{e}$ and $d_{n}$, except that $\alpha_{A_{\mu}}=-2.0$ (corresponding to $\mathrm{d}$ of Table III) and the curve with dashed line with triangles pointed up is a plot of the muon EDM $d_{\mu}$, which have all the same parameters for $d_{e}$ and $d_{n}$, except that $\left|A_{\mu}\right|=10.0$ and $\alpha_{A_{\mu}}=-2.5$ (corresponding to case $d$ of Table IV). 


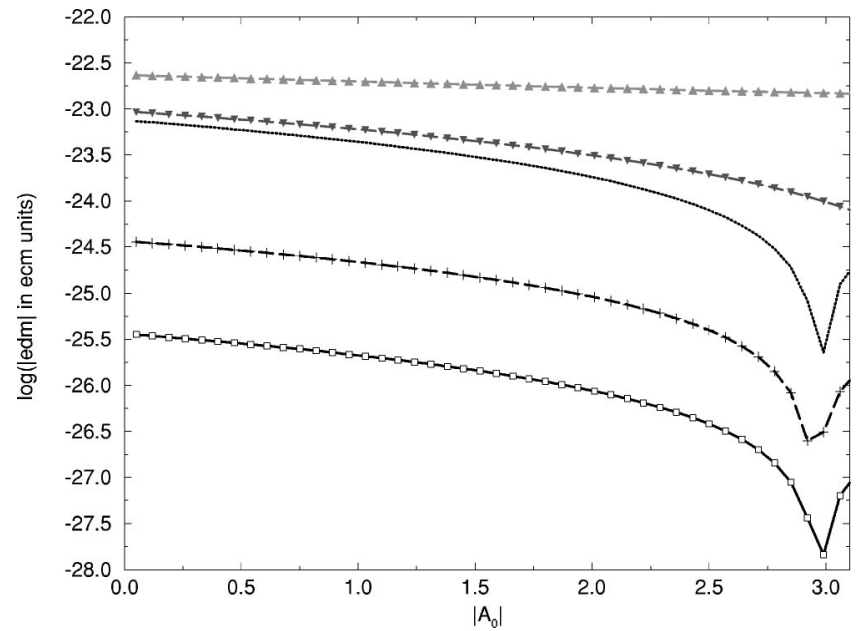

FIG. 5. A plot of the electron EDM $d_{e}$ (solid line with squares), of the neutron EDM $d_{n}$ (dashed line with plus signs), and of the muon EDM $d_{\mu}$ (dotted line) as a function of $\left|A_{0}\right|$ for the case when $\tan \beta=6, m_{0}=100, m_{1 / 2}=246, \quad \xi_{1}=.4, \quad \xi_{2}=-.77, \quad \xi_{3}=.55, \theta_{\mu}$ $=.4$, and $\alpha_{A_{e}}=1.15$, where all masses are in $\mathrm{GeV}$, corresponding to case e in Table I. The curve with dashed line with triangles pointed down, is a plot of the muon EDM $d_{\mu}$, which have all the same parameters as for $d_{e}$ and $d_{n}$, except that $\alpha_{A_{\mu}}=1.8$ (corresponding to e of Table III) and the curve with dashed line with triangles pointed up is a plot of the muon $\operatorname{EDM} d_{\mu}$, which have all the same parameters for $d_{e}$ and $d_{n}$, except that $\left|A_{\mu}\right|=7.0$ and $\alpha_{A_{\mu}}=2.5$ (corresponding to case e of Table IV).

error corridor constraint, as discussed at the end of the first paragraph in this section.

A plot of $d_{e}, d_{n}$ as a function of $\left|A_{0}\right|$ with the other parameters fixed by Table $\mathrm{I}$ is given in Figs. 1-5. Thus Fig. 1 contains case a of Table I, Fig. 2 contains case b of Table I etc. The three cases, 1, 2, and 3 for $d_{\mu}$ given by Tables II, III and IV are also plotted. These figures thus illustrate graphically the results discussed above. The analysis of Figs. 1-5 shows that the effect of nonuniversality is to shift the point of maximum cancellation between the chargino and the neutralino contributions for $d_{\mu}$, relative to what happens in $d_{e}$. This shift creates a much larger value for $d_{\mu}$ in the region where $d_{e}$ is compatible with the current experimental limits.

\section{CONCLUSION}

In this paper we have investigated the effect of nonuniversality in the trilinear soft parameter in the slepton sector on the scaling relation $d_{\mu} / d_{e} \simeq m_{\mu} / m_{e}$. We have shown that large violations of this relation can occur in the region of the parameter space, where the cancellation mechanism operates in the presence of nonuniversalities. Using this mechanism it is then shown that values of $d_{\mu}$, as much as a factor of 100 larger than implied by the scaling relation, can be obtained. Two branches of solutions were found. In the first solution the magnitudes $\left|A_{\mu}\right|$ and $\left|A_{e}\right|$ were assumed to be universal and the nonuniversalities arose only in the phases of $A_{e}$ and $A_{\mu}$, i.e., $\alpha_{A_{\mu}} \neq \alpha_{A_{e}}$. In this case, violations of scaling were generally modest but still sufficient to give $d_{\mu}$ of size $10^{-24} e \mathrm{~cm}$ consistent with Eqs. (1) and (3) and within reach of the sensitivity of the proposed BNL experiment [1]. In the second solution, nonuniversalities both in the magnitude and in the phase of the trilinear soft parameter were assumed, i.e., $\left|A_{\mu}\right| \neq\left|A_{e}\right|$ and $\alpha_{A_{e}} \neq \alpha_{A_{\mu}}$. In this case violations of the scaling formula were as much as a factor of 100 and one can get $d_{\mu} \sim O\left(10^{-23}\right) e \mathrm{~cm}$ consistent with Eqs. (1) and (3). Both of these possibilities are very encouraging for the observation of the muon EDM in the proposed Brookhaven experiment, which can explore the muon EDM at the level of $10^{-24} e \mathrm{~cm}$. Thus one may construe that the proposed Brookhaven experiment is an important probe of both $C P$ violation and of nonuniversalities in the muon sector.

\section{ACKNOWLEDGMENTS}

A communication from Yannis Semertzidiz, which led us to the present investigation, is acknowledged. This research was supported in part by NSF Grant No. PHY-9901057.
[1] Y.K. Semertzidiz et al., "Sensitive search for a permanent muon electric dipole moment," hep-ph/0012087.

[2] Y.K. Semertzidiz, in Frontier Tests of Quantum Electrodynamics and Physics of the Vacuum, edited by E. Zavattini, D. Bakalov, and C. Rizzo (1998), p. 369.

[3] J. Bailey et al., J. Phys. G 4, 345 (1978); J. Bailey et al., Nucl. Phys. B150, 1 (1979).

[4] F. Hoogeveen, Nucl. Phys. B341, 322 (1990); I.B. Khriplovich and M. Pospelov, Sov. J. Nucl. Phys. 53, 638 (1991).

[5] For a review, see S.M. Barr and W.J. Marciano, in CP Violation, edited by C. Jarlskog (World Scientific, Singapore, 1989), p. 455; W. Bernreuther and M. Suzuki, Rev. Mod. Phys. 63, 313 (1991).

[6] E. Commins et al., Phys. Rev. A 50, 2960 (1994); K. Abdullah et al., Phys. Rev. Lett. 65, 2347 (1990); P.G. Harris et al., ibid. 82, 904 (1999).

[7] See, e.g., J. Ellis, S. Ferrara, and D.V. Nanopoulos, Phys. Lett.
114B, 231 (1982); J. Polchinski and M.B. Wise, ibid. 125B, 393 (1983); E. Franco and M. Mangano, ibid. 135B, 445 (1984); M. Dugan, B. Grinstein, and L. Hall, Nucl. Phys. B255, 413 (1985); R. Garisto and J. Wells, Phys. Rev. D 55, 1611 (1997).

[8] P. Nath, Phys. Rev. Lett. 66, 2565 (1991); Y. Kizukuri and N. Oshimo, Phys. Rev. D 46, 3025 (1992).

[9] T. Ibrahim and P. Nath, Phys. Lett. B 418, 98 (1998); Phys. Rev. D 57, 478 (1998); 58, 019901 (1998); M. Brhlik, G.J. Good, and G.L. Kane, ibid. 59, 115004 (1999).

[10] T. Ibrahim and P. Nath, Phys. Rev. D 58, 111301 (1998).

[11] K.S. Babu, B. Dutta, and R.N. Mohapatra, Phys. Rev. D 61, 091701(R) (2000).

[12] C.Q. Geng and J.N. Ng, Phys. Rev. D 42, 1509 (1990).

[13] V. Barger, A. Das, and C. Kao, Phys. Rev. D 55, 7099 (1997).

[14] K.S. Babu, B. Dutta, and R.N. Mohapatra, Phys. Rev. Lett. 85, 5064 (2000). 
[15] K.S. Babu, S.M. Barr, and I. Dosner, Phys. Rev. D 64, 053009 (2001); hep-ph/0012303.

[16] Muon $(g-2)$ Collaboration, H.N. Brown et al., Phys. Rev. Lett. 86, 2227 (2001).

[17] T. Ibrahim, U. Chattopadhyay, and P. Nath, Phys. Rev. D 64, 016010 (2001).

[18] A. Czarnecki and W.J. Marciano, Nucl. Phys. B (Proc. Suppl.) 76, 245 (1999).

[19] D.A. Kosower, L.M. Krauss, and N. Sakai, Phys. Lett. 133B, 305 (1983); T.C. Yuan, R. Arnowitt, A.H. Chamseddine, and P. Nath, Z. Phys. C 26, 407 (1984).

[20] J.L. Lopez, D.V. Nanopoulos, and X. Wang, Phys. Rev. D 49, 366 (1994).

[21] U. Chattopadhyay and P. Nath, Phys. Rev. D 53, 1648 (1996); T. Moroi, ibid. 53, 6565 (1996); M. Carena, M. Giudice, and C.E.M. Wagner, Phys. Lett. B 390, 234 (1997); E. Gabrielli and U. Sarid, Phys. Rev. Lett. 79, 4752 (1997); K.T. Mahanthappa and S. Oh, Phys. Rev. D 62, 015012 (2000); T. Blazek, hep-ph/9912460; U. Chattopadhyay, D.K. Ghosh, and S. Roy, Phys. Rev. D 62, 115001 (2000).

[22] P. Nath and M. Yamaguchi, Phys. Rev. D 60, 116006 (1999).

[23] K. Agashe, N.G. Deshpande, and G.H. Wu, Phys. Lett. B 511, 85 (2001).

[24] SUGRA Working Group Collaboration, S. Abel et al., hep-ph/0003154.

[25] U. Chattopadhyay and P. Nath, Phys. Rev. Lett. 86, 5854 (2001).

[26] L. Everett, G.L. Kane, S. Rigolin, and L-T. Wang, Phys. Rev. Lett. 86, 3484 (2001).

[27] J. Feng and K.T. Matchev, Phys. Rev. Lett. 86, 3480 (2001).

[28] E.A. Baltz and P. Gondolo, Phys. Rev. Lett. 86, 5004 (2001).

[29] P. Nath and R. Arnowitt, Phys. Lett. B 336, 395 (1994); Phys. Rev. Lett. 74, 4592 (1995); F. Borzumati, M. Drees, and M. Nojiri, Phys. Rev. D 51, 341 (1995); H. Baer, M. Brhlik, D. Castano, and X. Tata, ibid. 58, 015007 (1998).

[30] T. Ibrahim and P. Nath, Phys. Rev. D 61, 095008 (2000); 62, 015004 (2000).

[31] For reviews, see P. Nath, R. Arnowitt, and A.H. Chamseddine, Applied $N=1$ Supergravity (World Scientific, Singapore, 1984); H.P. Nilles, Phys. Rep. 110, 1 (1984); H. Haber and G.L. Kane, ibid. 117, 75 (1985).

[32] P. Nath and R. Arnowitt, Phys. Rev. D 56, 2820 (1997); R. Arnowitt and P. Nath, in Perspectives on Supersymmetry, edited by G. Kane (World Scientific, Singapore, 1998), p. 442; hep-ph/9708254, and references therein.

[33] D. Chang, W-Y. Keung, and A. Pilaftsis, Phys. Rev. Lett. 82, 900 (1999); S.M. Barr and A. Zee, ibid. 65, 21 (1990). 